\title{
Foil Bearing Stiffness Estimation with Pseudospectral Scheme
}

\author{
Balaji Sankar, S Jana, and Sadanand Kulkarni \\ National Test Facility for Rolling Element Bearings, Propulsion Division, CSIR- National Aerospace Laboratories, INDIA
}

\begin{abstract}
Compliant foil gas lubricated bearings are used for the support of light loads in the order of few kilograms at high speeds, in the order of 50,000 RPM. The stiffness of the foil bearings depends both on the stiffness of the compliant foil and on the lubricating gas film. The stiffness of the bearings plays a crucial role in the stable operation of the supported rotor over a range of speeds. This paper describes a numerical approach to estimate the stiffness of the bearings using pseudo spectral scheme. Methodology to obtain the stiffness of the foil bearing as a function of weight of the shaft is given and the results are presented.
\end{abstract}

\section{Introduction}

Oil free turbo machinery technology is one in which the rotor supports do not employ oil lubrication like the conventional rolling element bearings. Turbo compressors, turbo generators and aircraft air-cycle machines already employ these novel rotor support systems. Of these support systems, self-acting gas foil bearings are increasingly being used for many low load and high speed applications [1]. The structure of the gas foil bearings is given in Fig 1.

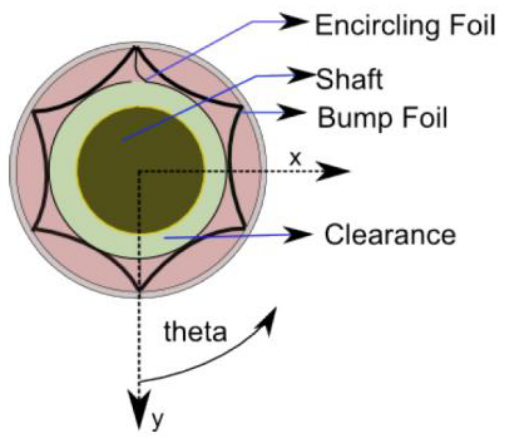

Figure 1. Front view of gas foil bearing [2]

In the gas foil bearing, the shaft is surrounded by the encircling foil. The encircling foil is supported by the bump foil. The bump foil rests on the firm foundation. There is a clearance between the rotating shaft and the encircling foil. Typically, the shaft is at an eccentric location in the bearing. As the shaft rotates, a convergent wedge is formed and the air is dragged into this wedge. As a result, there is a pressure increase in the convergent portion of the wedge. The shape of the edge depends on the location of the shaft in the bearing and on the deflection of the encircling foil due to the pressure force. When the force due to this pressure exceeds the shaft weight, the shaft levitates and rides on a film of air. More information regarding the structure and operation of the gas foil bearing is given in references [1-4].

Numerous publications have been made on the modelling of the pressure distribution in the foil bearing using multiple techniques. Bisson in Ref [5] gives an overview of conventional journal bearings and gas lubricated journal bearings. Ref [6] presents a comprehensive model covering the thermal energy transport in the bearing along with the rotor - bearing dynamics. Feng has presented an approach in which the bump foil of the bearing is modeled as a link spring structure in Ref [7]. A similar model has been used by Feng to study the behavior of the bearing in unbalanced conditions and unstable operation domains in Ref [8]. Piekos in Ref [9] has applied the pseudospectral scheme to journal bearings for micro gas turbines. This work and $\operatorname{Ref}[2,4$ and 10] are on the application of the scheme to compliant film gas foil bearing, where the deflection of the foil is also taken into account.

In this work, the gas foil bearing pressure distribution at a given operating condition is solved using the pseudo spectral scheme. By perturbing the eccentricity of the shaft and re-computing the pressure distribution, the change in force exerted by the bearing on the shaft is obtained. This change in force, when decomposed in $\mathrm{x}$ and $\mathrm{y}$ directions, gives us the $\mathrm{kx}$ and $\mathrm{ky}$ values of the bearing. The following section describes the governing equation and the solution procedure. The stiffness estimation for the chosen bearing configuration is presented next. The computed stiffness values are shown in the results section and concluded.

\section{Governing equations}

The simulation of the foil bearing involves iterative solution of the fluid model and structure model. The fluid model describes the pressure distribution in the fluid film 
surrounding the shaft. The structure model describes the deflection of the bump foil for a given pressure distribution in the encircling foil. An iterative solution is required because the computation of the pressure field depends on the film thickness, which in turn depends on the bump foil deformation. Detailed information on both the fluid and structure model is given in Ref [2]. A summary is given here for completeness of the paper.

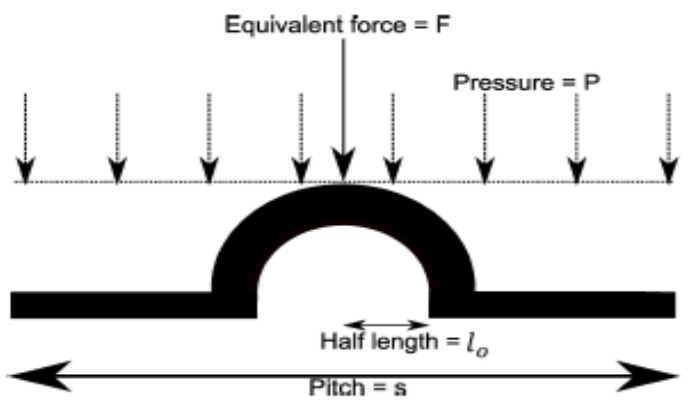

Figure 2. Pitch length of the bump foil

\section{Reynolds' equation}

The Reynolds' equation is given by

$$
\frac{\partial}{\partial x}\left(p h^{3} \frac{\partial p}{\partial x}\right)+\frac{\partial}{\partial y}\left(p h^{3} \frac{\partial p}{\partial y}\right)=6 \mu U\left\{\frac{\partial(p h)}{\partial x}\right\}
$$

In this equation, $\mathrm{p}:$ pressure at a location in film, $\mathrm{h}$ : Film thickness at a point in the film, $\mathrm{x}$ : Tangential coordinate, $y:$ Axial co-ordinate, $\mu$ : Viscosity of air and $\mathrm{U}$ : tangential surface speed of the shaft. Upon normalizing the quantities (same variables have been used for simplicity), the non-dimensional Reynolds' equation is given by

$$
\frac{\partial}{\partial x}\left(p h^{3} \frac{\partial p}{\partial x}\right)+\left(\frac{1}{(L / D)^{2}}\right) \frac{\partial}{\partial y}\left(p h^{3} \frac{\partial p}{\partial y}\right)=\Lambda \frac{\partial(p h)}{\partial x}(2)
$$

where $\mathrm{c}$ is the initial clearance, $\mathrm{R}$ is the radius of the bearing, pa is the ambient pressure and

$$
\text { Bearing Number }=\Lambda=\left(\frac{6 U R \mu}{C^{2} P_{A}}\right)
$$

Ambient pressure has been used for normalizing pressure, half length of the bearing for axial co-ordinate, radius of the bearing for the tangential co-ordinate and initial clearance of the bearing for film height.

\section{Structure model}

The structure model gives the film thickness distribution in the bearing for a given shaft location and pressure distribution. It is adapted from [11]. Multiple models describing the deflection of the bump foil structure are available. This model is chosen due to its simplicity. The non-dimensional film thickness in the foil bearing is given by

$$
h=1+e * \cos \left(\theta-\phi_{o}\right)+\alpha(p-1)
$$
is

The non-dimensional compliance used in the equation

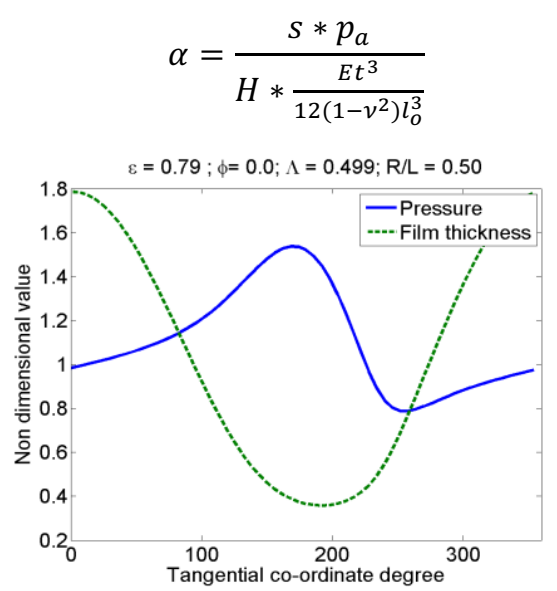

Figure 3 Pressure and film thickness distribution

where, $\mathrm{t}$ is the thickness of the bump foil, $\mathrm{s}$ is the pitch length of the bump foil, lo is the half length of the bump foil, $l_{o}$ is the Poisson's ratio and $\mathrm{E}$ is the Young's modulus of the material. The pitch length and half-length are shown in Fig. 2.

\section{Solution procedure}

Detailed procedure on using the pseudo spectral scheme to solve the loosely coupled system of equations presented here is given in Ref[10]. A brief summary is given here. An initial pressure distribution is first assumed and the film thickness is calculated for this pressure distribution. The dependent variables are expressed as a series, in terms of Fourier series in tangential direction and as Chebyshev's series in the axial direction. Writing the dependents in the form of these series, enables us to estimate their derivatives using global approximations. On the contrary, using finite difference techniques to estimate the derivatives uses local approximation. Once spatial derivatives are estimated using series expansions, a time marching scheme is used to estimate the pressure distribution, while fixing the shaft location at a given eccentricity and attitude angle. The time marching is carried with recursive solving of the pressure and film thickness distribution until a steady state solution of the pressure distribution is reached. The same validated code is used for stiffness estimation in this work.

\section{Stiffness estimation}

Consider a bearing number of $\Lambda=0: 4985$, radius of 19.05 $\mathrm{mm}$ and $\frac{R}{L}=\frac{1}{2}$. For this configuration, the nondimensional pressure and film thickness distribution are shown in Fig. 3. To visualize the pressure distribution and forces better, the plot is made in polar co-ordinates for the same configuration in Fig. 4. In this plot, the blue circle represents the location of the shaft and arrow mark shows the direction of the force and the magnitude. As the attitude increases from 0 degrees, the force 
increasingly becomes vertical and at a certain attitude (approximately 47 degrees), the force becomes fully vertical and supports the weight of the shaft without any horizontal force. Fig. 5 shows variation of forces with attitude angle. Only when the vertical force is equal to shaft weight and there is no horizontal force, stationary position of the shaft is theoretically possible.

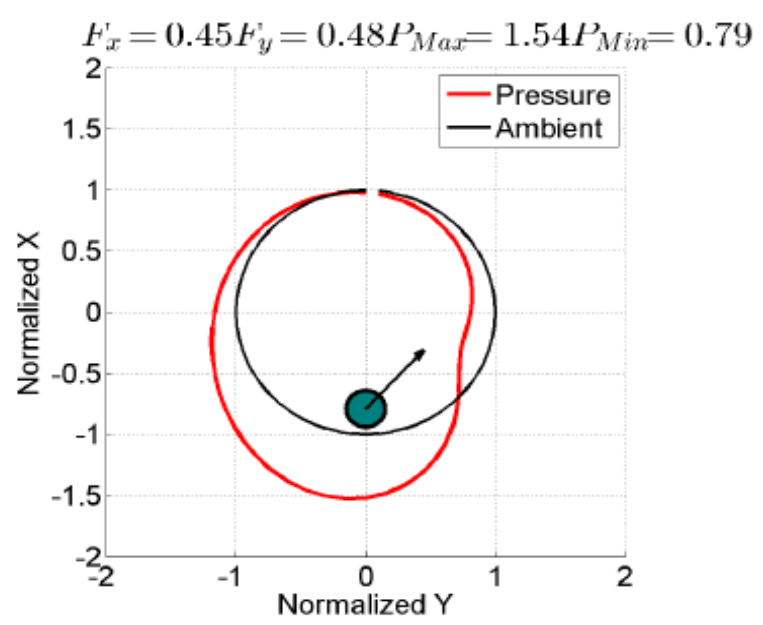

Figure 4. Polar plot of pressure distribution, $\phi=0$ degrees, $\varepsilon=$ 0.79

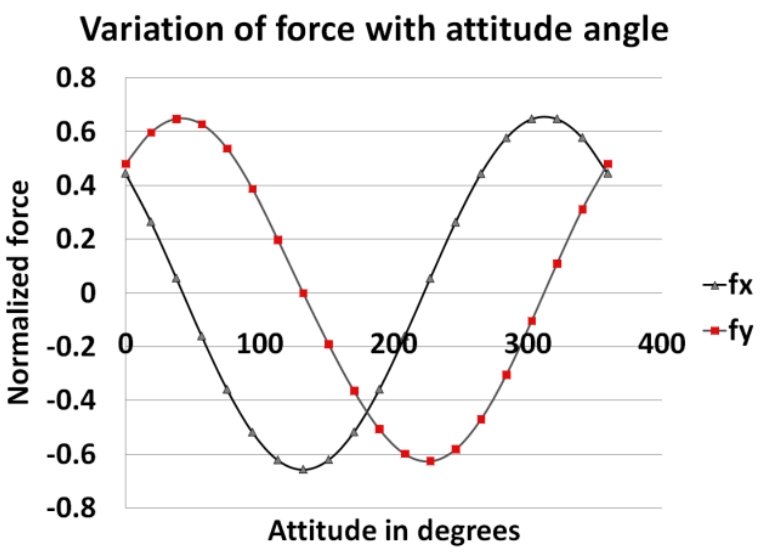

Figure 5. Variation of forces with attitude angle

Table 1. Attitude and eccentricity for different loads

\begin{tabular}{|c|c|c|c|}
\hline S No & load (N) & Epsilon & Phi \\
\hline 1 & 9.9 & 0.19 & 72.2 \\
\hline 2 & 15 & 0.27 & 69.8 \\
\hline 3 & 20 & 0.35 & 67.2 \\
\hline 4 & 24.9 & 0.43 & 64 \\
\hline 5 & 29.9 & 0.51 & 60.6 \\
\hline 6 & 35 & 0.59 & 56.6 \\
\hline 7 & 39.9 & 0.66 & 52.6 \\
\hline 8 & 45 & 0.73 & 48.1 \\
\hline 9 & 49.9 & 0.79 & 44 \\
\hline
\end{tabular}

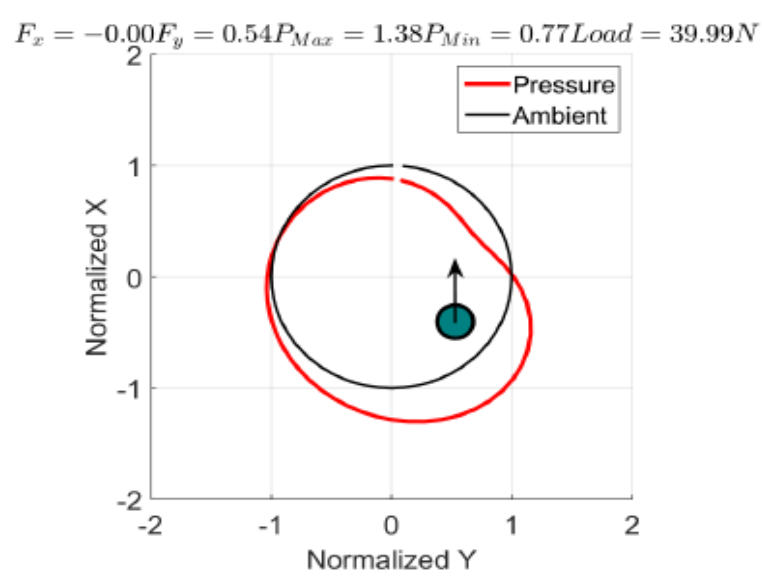

Figure 6. Balanced location for $40 \mathrm{~N}$ load

In this work the stiffness calculation is done for a range of load values at various eccentricities and attitude angles. In order to estimate the stiffness at a particular load, the eccentricity and attitude angle for that load are found first. The eccentricity and attitude angles for various loads are shown in table I and shown pictorially for a few load levels in Fig 6, and 7. After estimating the eccentricity and attitude for a given load, the eccentricity value is slightly increased by $1 \%$ while keeping the attitude same. This results in a change of $x$ coordinate of the shaft, $\mathrm{y}$ co-ordinate of the shaft and the forces in the $\mathrm{x}$ and $y$ direction. From the changes in co-ordinates of the shaft and changes in force, the 4 stiffness values are calculated as given in Table II.

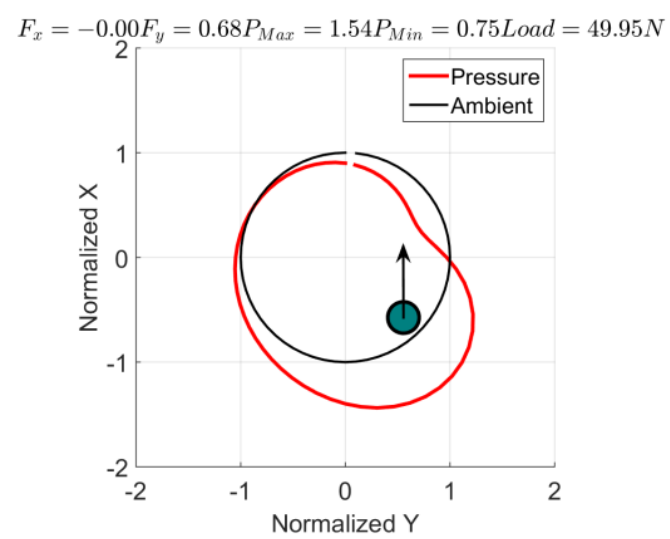

Figure 7 Balanced location for 50 Newtons

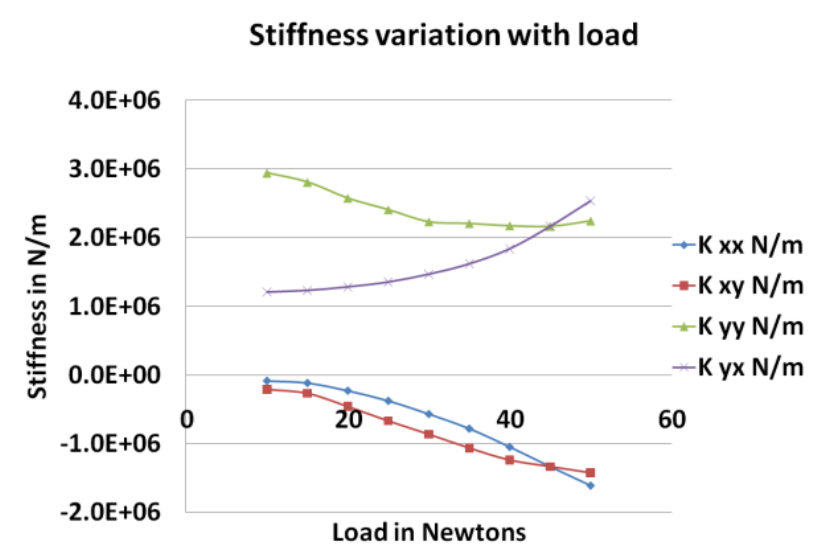

Figure 8 Stiffness variation with load 
Table 2. Stiffness calculation

\begin{tabular}{|l|l|l|l|l|l|l|l|l|l|l|l|}
\hline Epsilon & Fy & DFX & DFY & X & Y & DX & DY & K xx & Ky & K yy & K yx \\
\hline & N & Newton & Newton & micron & micron & micron & micron & N/m & N/m & N/m & N/m \\
\hline 0.18 & 10 & $-7.35 \mathrm{e}-3$ & $1.03 \mathrm{E}-01$ & $8.30 \mathrm{E}-06$ & $3.54 \mathrm{e}-06$ & $8.50 \mathrm{e}-8$ & $3.50 \mathrm{e}-8$ & $-8.65 \mathrm{e}+04$ & $-2.10 \mathrm{e}+05$ & $2.94 \mathrm{e}+6$ & $1.21 \mathrm{e}+6$ \\
\hline 0.27 & 15 & $-1.47 \mathrm{e}-2$ & $1.54 \mathrm{e}-1$ & $1.23 \mathrm{e}-05$ & $5.62 \mathrm{e}-06$ & $1.25 \mathrm{e}-7$ & $5.50 \mathrm{e}-8$ & $-1.18 \mathrm{e}+05$ & $-2.67 \mathrm{e}+05$ & $2.81 \mathrm{e}+6$ & $1.24 \mathrm{e}+6$ \\
\hline 0.36 & 20 & $-3.68 \mathrm{e}-2$ & $2.06 \mathrm{e}-1$ & $1.60 \mathrm{e}-05$ & $8.05 \mathrm{e}-06$ & $1.60 \mathrm{e}-7$ & $8.00 \mathrm{e}-8$ & $-2.30 \mathrm{e}+05$ & $-4.60 \mathrm{e}+05$ & $2.57 \mathrm{e}+6$ & $1.29 \mathrm{e}+6$ \\
\hline 0.44 & 25 & $-7.35 \mathrm{e}-2$ & $2.65 \mathrm{e}-1$ & $1.93 \mathrm{e}-05$ & $1.09 \mathrm{e}-05$ & $1.95 \mathrm{e}-7$ & $1.10 \mathrm{e}-7$ & $-3.77 \mathrm{e}+05$ & $-6.69 \mathrm{e}+05$ & $2.41 \mathrm{e}+6$ & $1.36 \mathrm{e}+6$ \\
\hline 0.53 & 30 & $-1.25 \mathrm{e}-1$ & $3.24 \mathrm{e}-1$ & $2.21 \mathrm{e}-05$ & $1.43 \mathrm{e}-05$ & $2.20 \mathrm{e}-7$ & $1.45 \mathrm{e}-7$ & $-5.68 \mathrm{e}+05$ & $-8.62 \mathrm{e}+05$ & $2.23 \mathrm{e}+6$ & $1.47 \mathrm{e}+6$ \\
\hline 0.61 & 35 & $-1.91 \mathrm{e}-1$ & $3.97 \mathrm{e}-1$ & $2.43 \mathrm{e}-05$ & $1.81 \mathrm{e}-05$ & $2.45 \mathrm{e}-7$ & $1.80 \mathrm{e}-7$ & $-7.80 \mathrm{e}+05$ & $-1.06 \mathrm{e}+6$ & $2.21 \mathrm{e}+6$ & $1.62 \mathrm{e}+6$ \\
\hline 0.68 & 40 & $-2.72 \mathrm{e}-1$ & $4.78 \mathrm{e}-1$ & $2.58 \mathrm{e}-05$ & $2.22 \mathrm{e}-05$ & $2.60 \mathrm{e}-7$ & $2.20 \mathrm{e}-7$ & $-1.05 \mathrm{e}+6$ & $-1.24 \mathrm{e}+6$ & $2.17 \mathrm{e}+6$ & $1.84 \mathrm{e}+6$ \\
\hline 0.75 & 45 & $-3.53 \mathrm{e}-1$ & $5.74 \mathrm{e}-1$ & $2.66 \mathrm{e}-05$ & $2.64 \mathrm{e}-05$ & $2.65 \mathrm{e}-7$ & $2.65 \mathrm{e}-7$ & $-1.33 \mathrm{e}+6$ & $-1.33 \mathrm{e}+6$ & $2.16 \mathrm{e}+6$ & $2.16 \mathrm{e}+6$ \\
\hline 0.81 & 50 & $-4.34 \mathrm{e}-1$ & $6.84 \mathrm{e}-1$ & $2.69 \mathrm{e}-05$ & $3.04 \mathrm{e}-05$ & $2.70 \mathrm{e}-7$ & $3.05 \mathrm{e}-7$ & $-1.61 \mathrm{e}+6$ & $-1.42 \mathrm{e}+6$ & $2.24 \mathrm{e}+6$ & $2.53 \mathrm{e}+6$ \\
\hline
\end{tabular}

\section{Results}

The variation of the 4 stiffness components as the weight of the shaft is increase is shown in Fig. 8.The negative stiffness in the $\mathrm{x}$ direction is due to the increased suction pressure to the right side of the shaft, as the gap between the shaft and encircling foil decreases. Increase in load causes higher eccentricity of the shaft, as given in table I. It is also seen that absolute value of the stiffness of all 4 components increase with increase in load. This increase in stiffness with increase in load alters the natural frequencies of the system. This change in natural frequencies must be accounted for while designing the system for the given operating condition.

\section{References}

1. C. DellaCorte. Tribological limitations in gas turbine engines a workshop to identify the challenges and set future directions. Technical report,NASA, (2000).

2. BalajiSankar. Numerical simulation and experimental validation of compliant surface gas film bearings for high speed applications, Master's thesis, Academy of Scientific and Innovative Research, (2012).

3. A.Cameron. Basic Lubrication Theory.Longman, (1971).

4. Balaji Sankar and SadanandKulkarni.Non linear time dependent simulations of high speed gas lubricated bearings-an object oriented model,presented in International conference on advanced research in mechanical engineering, ICARME-2012, (2012).

5. Edmond $\mathrm{E}$ Bisson and William $\mathrm{J}$ Anderson. Advanced bearing technology, Nasa sp-38. NASA Special Publication, 38, (1964).

6. Luis San Andreas, Thermohydrodynamic analysis of bump type gas foil bearings: A model anchored to test data. Journal of Engineering for Gas Turbines and Power, 132:042504-1 to042504-10, (2010).

7. Kai Feng. Analytical model of bump-type foil bearings using a linkspring structure and a finiteelement shell model. Journal of Tribology, 132:0210706-1 to 0210706-11, (2010).

8. Kai Feng. Parametric studies on static performance and nonlinear instability of bump-type foil bearings. Journal of System Design and Dynamics, 6, (2010).

9. Edward Stanley Piekos.Numerical Simulation of Gas-Lubricated Journal Bearings for Microfabricated Machines.PhD thesis, MIT, (2000).

10. BalajiSankar and SadanandKulkarni.Modeling of gas lubricated compliant foil bearings using pseudo spectral scheme. Tribology Online, 10(5), pp. 295305, (2015).

11. Z.C. Peng and M.M. Khonsari, Hydrodynamic analysis of compliant foil bearings with compressible air flow. Transactions of the ASME, 126:542-546, (2004). 